Check for updates

Cite this: J. Mater. Chem. A, 2020, 8 , 1279

Received 29th September 2019 Accepted 13th December 2019

DOI: $10.1039 / c 9 t a 10779 g$

rsc.li/materials-a

\section{Synergy of co-sensitizers in a copper bipyridyl redox system for efficient and cost-effective dye- sensitized solar cells in solar and ambient light $\uparrow$}

\begin{abstract}
Ellie Tanaka, (D) ${ }^{a}$ Hannes Michaels, (ID ${ }^{b}$ Marina Freitag (iD t $^{* b}$ and Neil Robertson (ID *a
Dye-sensitized solar cells (DSSCs) have a great potential to expand the area of photovoltaics towards portable or indoor applications. Since the dye is one of the most costly components in the device, it is always of interest to find ways to lower its associated cost. Here, we propose the co-sensitization technique as a simple and effective solution towards this aim. A less-expensive $\pi-A$ dye (5T) was mixed with a better-performing $D-A-\pi-A$ dye (XY1) to fabricate the co-sensitized devices $(X Y 1+5 T)$. The dyes were combined with a $\mathrm{Cu}^{1 / 1 /}(\mathrm{tmby})_{2}$ (tmby $=\left(4,4^{\prime}, 6,6^{\prime}\right.$-tetramethyl-2, $2^{\prime}$-bipyridine)) electrolyte and tested at 1 sun and 0.1 sun conditions. The XY1 + 5T devices showed similar power conversion efficiency (PCE) to that of the XY1-only devices at 1 sun (avg. 9.1\%), and superior PCE at 0.1 sun (avg. 9.4\% vs. 8.6\%). The charge transport behaviour in the devices was investigated through a combination of photophysical measurements, which revealed complementary effects of both dyes during device operation. The performance of XY1 + 5T at lower light intensity was further assessed using artificial fluorescent lighting, which marked a PCE as high as $29.2 \%$ at 1000 lux, among the highest reported. Finally, the cost of dye was estimated to be reduced by ca. 30\% if an XY1-only device is replaced by XY1 + 5T with no loss in efficiency. The cost-performance is thus improved 1.4 times. Our findings will revalue the cosensitization method as a means to address the challenges of DSSC commercialization.
\end{abstract}

\section{Introduction}

Photovoltaic energy generation is in growing demand to tackle the world population growth and future energy shortage. ${ }^{1-3}$ While crystalline silicon-based solar cells are well established for capturing outdoor sunlight, their performance is poor under low light conditions due to the indirect bandgap of crystalline silicon. ${ }^{4-6}$ Therefore, the development of alternative technologies is needed for areas such as portable charging devices or indoor-light capturing. Dye-sensitized solar cells (DSSCs) are expected to meet this category of photovoltaic applications, not only due to their ease of fabrication, but also based on recent reports which reveal their superior performance to any other established photovoltaic technology when tested in indoor-light conditions. ${ }^{7-9}$

The DSSC is assembled from multiple components, namely the transparent conductive oxide window, ${ }^{10-13}$ n-type oxide, ${ }^{14-17}$

${ }^{a}$ School of Chemistry, University of Edinburgh, Kings Buildings, Edinburgh, EH9 3FJ, UK. E-mail: neil.robertson@ed.ac.uk

${ }^{b}$ Department of Chemistry, Angström Laboratory, Uppsala University, Box 523, Uppsala, SE-751 20, Sweden. E-mail: marina.freitag@kemi.uu.se

$\dagger$ Electronic supplementary information (ESI) available. See DOI: 10.1039/c9ta10779g

\$ Current address: Chemistry, School of Natural and Environmental Science, Newcastle University, Newcastle upon Tyne, UK. dye ${ }^{18-21}$ electrolyte ${ }^{22-25}$ and cathode. ${ }^{26-29}$ Dedicated investigation on each component is the key towards optimization of the device as a whole. ${ }^{30}$ The present study will particularly focus on the dye, the core component for visible light capture in a DSSC. Although the dye uptake per device is relatively low $\left(\sim 10^{-8} \mathrm{~mol}\right.$ $\mathrm{cm}^{-2}$ ), the commercial price of dyes could rise to an order of magnitude higher than the simple materials-based synthesis cost, $\S$ likely due to additional labour costs of batch synthesis. Obviously, complex multistep reactions will result in increased materials and labour costs giving limitations to mass production. Reducing the cost of the dye is thus crucial for the reduction of the total cost of the device.

Evolving from the classic ruthenium-centred dyes, organic dyes free from scarce metals have become the dye design trend in the past few years. Owing to their distinctively higher absorption coefficients in respect to metal-centred dyes, relatively high efficiencies have been achieved with thinner $\mathrm{TiO}_{2}$ films. Thus, the shift to metal-free organic dyes is an initial step to lower the overall cost of the sensitizer in the device. Nonetheless, the highest performing organic dyes tend to have a more complex structure and hence higher cost. ${ }^{20,23,31}$ A simple and versatile way to deal with this challenge is by co-sensitizing the target dye with another less expensive dye. The technique

$\S$ Claim based on our synthesis cost analysis and private conversation with suppliers. 
has been widely adopted to achieve panchromatic absorption by complementing the absorption spectrum of each individual dye. , $, 9,20,23,31-37$ However, its potential towards reducing the overall device cost has been rarely discussed until now.

Most reported sensitizers in DSSCs have the molecular structure of D- $\pi-\mathrm{A}$ (donor/conjugated spacer/acceptor), where other variations include $\mathrm{D}-\mathrm{A}-\pi-\mathrm{A}$ as well as the "donor-free" $\pi-$ A. The donor or end- $\pi$ moiety directly interacts with the electrolyte whereas the end acceptor moiety chemically binds to the surface of the mesoporous $\mathrm{TiO}_{2}$. XY1 (Fig. 1(a)), one of the earliest $\mathrm{D}-\mathrm{A}-\pi-\mathrm{A}$ dyes developed by Zhang et al. ${ }^{38}$ is a deep purple dye with a molar extinction coefficient of $56500 \mathrm{M}^{-1} \mathrm{~cm}^{-1}$ at $552 \mathrm{~nm}$. The structure employs a bulky donor in order to inhibit undesired dye aggregation and minimize charge recombination. The dye has been proven to show good power conversion efficiency (PCE) in DSSCs with a range of electrolytes including the $\mathrm{Cu}^{\mathrm{I} / \mathrm{II}}(\mathrm{tmby})_{2}$ (tmby $=4,4^{\prime}, 6,6^{\prime}$-tetramethyl-2,2'-bipyridine) redox couple. ${ }^{\mathbf{8}, 38}$ On the other hand, 5T (Fig. 1(b)), one of the earliest $\pi-\mathrm{A}$ dyes introduced by Abate et al.,$^{39,40}$ is a red-orange dye with a molar extinction coefficient of $39000 \mathrm{M}^{-1} \mathrm{~cm}^{-1}$ at $478 \mathrm{~nm} .^{41}$ The dye has been studied in combination with $\mathrm{I}^{-} / \mathrm{I}_{3}{ }^{-}$and $\mathrm{Co}^{\mathrm{II} / \mathrm{III}}(\mathrm{bpy})_{3}$ (bpy $=2,2^{\prime}$-bipyridine) redox electrolytes to give promising efficiencies $(\mathrm{PCE}=$ 7.6\% $\left(\mathrm{I}^{-} / \mathrm{I}_{3}{ }^{-}\right)$and $\left.9.0 \%\left(\mathrm{Co}^{\mathrm{II} / \mathrm{III}}(\mathrm{bpy})_{3}\right)\right) . .^{\mathbf{4 1 , 4 2}}$

The main issue with XY1 is the costly synthesis which involves 12 steps in total, among which 3 steps require $\mathrm{Pd}\left(\mathrm{PPh}_{3}\right)_{4}$ at $\$ 28$ per $\mathrm{g}$ (Fig. $\mathrm{S} 1 \dagger$ ). Our materials-based cost estimation returns the overall synthesis cost of XY1 to be as high as $\$ 868$ per mmol ( $\$ 537$ per g) (Table $\mathbf{S} 2 \dagger$ ), which is already higher than the commercial price of the classic N719 dye, which is $\$ 632$ per mmol (\$532 per g) $9 . .^{\mathbf{4 3}}$ Therefore, it would be of interest to find a way to reduce the cost of the sensitizer while maintaining the overall power output. Here, we propose $5 \mathrm{~T}$ as a co-sensitizer candidate for $\mathrm{XY} 1$ in the $\mathrm{Cu}^{\mathrm{I} / \mathrm{II}}(\mathrm{tmby})_{2}$ system based on three points (Fig. 2): (1) the UV-vis absorption peak ranges are complementary, ${ }^{38,41}(2)$ the ground-state potential of $5 \mathrm{~T}(1.08 \mathrm{~V})$ is more positive than the redox potential of $\mathrm{Cu}^{\mathrm{I} /}$ ${ }^{\mathrm{II}}$ (tmby) $(0.87 \mathrm{~V})^{44}$ and (3) the synthesis is relatively simple (9 steps in total, where many of them are repetitive).

The effect of the choice of redox mediator upon the $V_{\mathrm{OC}}$ of $5 \mathrm{~T}$ has been previously demonstrated when a $\mathrm{Co}^{\mathrm{II} / \mathrm{III}}(\mathrm{bpy})_{3}$ electrolyte was used instead of $\mathrm{I}^{-} / \mathrm{I}_{3}{ }^{-}$, where the $V_{\mathrm{OC}}$ improved from $0.62 \mathrm{~V}$ to $0.79 \mathrm{~V} \cdot{ }^{42}$ Further increase in $V_{\mathrm{OC}}$ is expected by pairing the dye with $\mathrm{Cu}^{\mathrm{I} / \mathrm{II}}(\mathrm{tmby})_{2} \cdot \mathrm{Cu}^{\mathrm{I} / \mathrm{II}}(\mathrm{tmby})_{2}$ belongs to a family of recently developed fast electron-transfer copper-centred redox mediators that have opened up a new path in DSSC research. ${ }^{45-47}$ They not only have deep redox potentials but also allow minimal driving force $(\sim 0.1 \mathrm{~V})$ for dye regeneration, greatly reducing the overpotential losses at the electrolyte/dye interface. ${ }^{\mathbf{4 4 8}}$ They are an interesting electrolyte choice especially if further interests lie in solid-state DSSCs (which is out of the scope of this report). ${ }^{\mathbf{4 5 , 4 6 , 4 8}}$

The DSSCs fabricated in this study were sensitized with three dye conditions; (1) XY1 only (XY1), (2) 5T only (5T) and (3)

ๆ $£ 416$ per $\mathrm{g}$. Converted to USD by $1 \mathrm{GBP}=1.28$ USD.

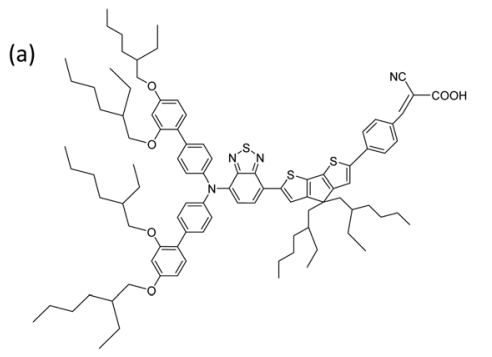

(b)

Fig. 1 Molecular representation of (a) XY1 dye and (b) 5T dye used in this study.

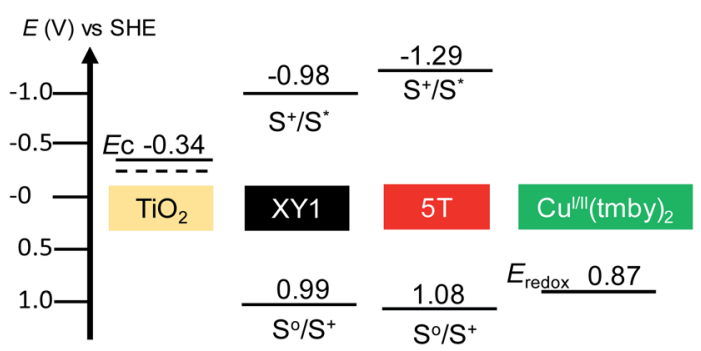

Fig. 2 Energy diagram of the dyes used in this study, in comparison with the $\mathrm{TiO}_{2}$ anode and $\mathrm{Cu}^{\mathrm{I} / I}(\text { tmby) })_{2}$ electrolyte. All state levels are described in volts. The values were taken from the literature. ${ }^{38,41,48}$

a combination of $5 \mathrm{~T}$ and XY1 (XY1 + 5T). All solar cells were assembled with the $\mathrm{Cu}^{\mathrm{I} / \mathrm{II}}(\mathrm{tmby})_{2}$ electrolyte. The currentvoltage performance of the devices were characterized in response to 1 sun (AM1.5G) and 0.1 sun, and further insights on the dye/electrolyte behaviour were gained by photophysical measurements including electron lifetime and transient absorption spectroscopy.

In brief, the $5 \mathrm{~T}$ dye in combination with $\mathrm{Cu}^{\mathrm{I} / \mathrm{II}}\left(\mathrm{tmby}_{2}\right.$ electrolyte attained a $V_{\mathrm{OC}}$ of max. $0.99 \mathrm{~V}$ (avg. $0.97 \mathrm{~V}$ ), notably higher than the previously reported values with $\mathrm{I}^{-} / \mathrm{I}_{3}{ }^{-}$and $\mathrm{Co}^{\mathrm{II} / \mathrm{III}}$ electrolyte. ${ }^{41,42}$ Studies on the co-sensitized XY1 $+5 \mathrm{~T}$ devices suggested that XY1 and 5T mutually support each other at the different stages of charge transport within the cell cycle. As a consequence, the XY1 + 5T device exhibited a PCE of max. 9.53\% (avg. 9.1\%) at 1 sun, comparable to the XY1-only device (max. 9.56\%, avg. 9.1\%). At lower light intensities such as 0.1 sun, the PCE rose to max. 10.2\% (avg. 9.4\%), surpassing the XY1 (max. 8.94\%, avg. 8.6\%). Photophysical measurements suggested that the co-sensitized devices have better dye coverage compared to the mono-sensitized devices, as well as longer electron lifetime especially at lower light intensities. These indications were further assessed by testing XY1 $+5 \mathrm{~T}$ under indoor-light conditions; revealing a high PCE of max. 29.2\% (avg. 28\%) at 1000 lux. Although XY1 shows higher performance than $5 \mathrm{~T}$ in mono-sensitized DSSCs, the co-sensitized devices appear to contain more 5T dye than XY1 dye to achieve a similar PCE to the XY1-only DSSC. From UV-vis spectra analysis, the adsorbed amount of $5 \mathrm{~T}$ dye in $\mathrm{XY} 1+5 \mathrm{~T}$ is estimated to be 1.7 times larger than the XY1 dye. As a result, the total dye cost (per area and per PCE) in XY1 $+5 \mathrm{~T}$ is estimated to be lowered by $c a$. 
$30 \%$ in respect to the XY1-only device. We believe our studies are the first of a kind to evaluate the co-sensitization technique from a cost-related viewpoint.

\section{Experimental section}

\section{Materials preparation and device fabrication}

Materials. Reagents were purchased from Merck and used without further purification, unless otherwise specified. 5T was synthesized according to the literature. ${ }^{39} \mathrm{XY} 1$ dye, $\mathrm{Cu}^{\mathrm{I}}(\text { tmby })_{2}(-$ TFSI) (TFSI $=$ trifluoromethanesulfonimide) and $\mathrm{Cu}^{\mathrm{II}}(\text { tmby })_{2}(-$ TFSI $)_{2}$ were purchased from Dyenamo $A B$ and used without further purification. 4-tert-Butyl pyridine $(t \mathrm{BP})$ was purchased from Merck and distilled before use.

DSSC fabrication. Fluorine-doped tin oxide (FTO) conductive glass (NSG TEC ${ }^{\text {TM }}$ 15, Pilkington) was pre-cut and cleaned by sonication in a $2 \%$ aqueous detergent solution (Hellmanex, Hellma) and ethanol, followed by treatment with $\mathrm{UV} / \mathrm{O}_{3}$ for 15 min. The $\mathrm{TiO}_{2}$ blocking layer was formed on the FTO substrate by immersion in $40 \mathrm{mM} \mathrm{TiCl}_{4}$ aq. at $70{ }^{\circ} \mathrm{C}$. This procedure was applied twice. The mesoporous $\mathrm{TiO}_{2}$ layer was formed by screen-printing 30NR-D $(4 \mu \mathrm{m})$ and WER2-0 $(4 \mu \mathrm{m}) \|$ (both pastes from GreatCell Solar), followed by annealing at $450{ }^{\circ} \mathrm{C}$ for $30 \mathrm{~min}$. The mesoporous films were treated with $13 \mathrm{mM} \mathrm{TiCl}_{4}$ aq. at $70{ }^{\circ} \mathrm{C}$ and annealed at $450{ }^{\circ} \mathrm{C}$ for $30 \mathrm{~min}$. The films were introduced into the dye bath while they were still warm $\left(60-80{ }^{\circ} \mathrm{C}\right)$ and left at r.t. overnight. The XY1 dye bath consisted of $0.1 \mathrm{mM}$ XY1 dye in ethanol/chloroform $7: 3(\mathrm{v} / \mathrm{v})$ with $1 \mathrm{mM}$ chenodeoxycholic acid (CDCA). The 5T dye bath consisted of $0.1 \mathrm{mM} 5 \mathrm{~T}$ dye in ethanol/chloroform $7: 3(\mathrm{v} / \mathrm{v})$ with $0.4 \mathrm{mM}$ CDCA. For the pure XY1 and 5T samples, $4 \mathrm{~mL}$ of the premade dye solution was poured in a vial with warm films. For the XY1 + 5T co-sensitized samples, $2 \mathrm{~mL}$ of the premade $5 \mathrm{~T}$ solution was poured in a vial with the warm films, immediately followed by addition of $2 \mathrm{~mL}$ of the XY1 solution $(\mathrm{XY} 1 / 5 \mathrm{~T}=1: 1, \mathrm{~mol} / \mathrm{mol}$ and $\mathrm{v} / \mathrm{v})$. The vial was kept still to allow the two dye solutions to mix together in the bath through natural convection.

Poly(3,4-ethylenedioxythiophene) (PEDOT) was electropolymerized onto a FTO substrate (TEC8, Pilkington) from an aqueous solution of $0.1 \mathrm{M}$ sodium dodecyl sulfate and $0.01 \mathrm{M}$ ethylenedioxythiophene (EDOT). ${ }^{29}$ Subsequently, the photoanodes were assembled with a PEDOT cathode using a UVcuring glue (TB3035B UV Curing Sealant, Threebond) and a CS2010 High Power UV curing LED system (Thorlabs). ${ }^{9}$ The electrolyte was injected through a predrilled hole in the cathode and the hole was sealed with the UV-curing glue. The composition of the electrolyte was $0.2 \mathrm{M} \mathrm{Cu}^{\mathrm{I}}$ (tmby) $)_{2}$ (TFSI), $0.06 \mathrm{M}$ $\mathrm{Cu}^{\mathrm{II}}$ (tmby)(TFSI) $)_{2}, 0.1 \mathrm{M}$ lithium bis(trifluoromethanesulfonyl) imide (LiTFSI) and 0.6 $\mathrm{M} t \mathrm{BP}$ in anhyd. acetonitrile. Finally, silver paint (Hans-Wohlbring $\mathrm{GmbH}$ ) was applied to the contacts to allow optimum conductivity.

For indoor-light testing, larger-area DSSCs (active area 3.20 $\mathrm{cm}^{2}$ ) were fabricated following the method described above.

|| The film thickness was measured with a profilometer (data not shown).

\section{Characterization methods}

UV-vis absorption measurements. The UV-vis absorption was measured on an HR-2000 spectrophotometer (Ocean Optics) with DHL-2000-BAL (Mikropack) as light source. For the samples, $4 \mu \mathrm{m}$-thick transparent $\mathrm{TiO}_{2}$ films (30NR-D) deposited on FTO were prepared following a similar procedure as for the films for solar cells. The films were then immersed in the designated $0.25 \mathrm{mM}$ dye bath (4-times diluted stock $0.1 \mathrm{mM}$ dye solution) overnight. The samples were referenced to a nonsensitized $\mathrm{TiO}_{2} /$ FTO film and were illuminated from the film side.

Photocurrent-voltage measurements $(J-V)$. The $J-V$ characteristics were measured with a class AAA SINUS-220 solar simulator (Wavelabs) connected to a Solar Cell I-V Test System (Ossila). The light source was a light-emitting diode (LED) white light, equipped with a self-calibration system to AM1.5G standard spectra ( 1 sun) or AM $1.5 \mathrm{G}$ at $10 \%$ intensity ( 0.1 sun) where indicated. The active area of each cell was fixed to $0.160 \mathrm{~cm}^{2}$ with a thin black metal mask. In the case of indoor-light testing, an Osram Warm White 930 white fluorescent lamp (18 W) was used as the illumination source. The active area of each cell was $3.20 \mathrm{~cm}^{2}$ and the light intensity was calibrated to $1000 \mathrm{lux}$ (303.1 $\mu \mathrm{W} \mathrm{cm}^{-2}$ ) using a lux meter (TES 1334). The current-voltage scans were then recorded using a PGSTAT 100 potentiostat (Autolab).

Incident photon-to-current conversion efficiency (IPCE). IPCE measurements were performed as previously described. ${ }^{\mathbf{4 7}}$ The samples were illuminated with an ASB-XE-175 xenon light source (Spectral Products) monochromated through a CM110 monochromator (Spectral Products) from the photoanode side. The background was calibrated with a silicon reference cell (Fraunhofer) before the actual measurement. The integrated photocurrent was calculated from the IPCE spectra based on ASTM G173-03 reference spectra derived from SMARTS v. 2.9.2.

Time-resolved 'toolbox' measurements. Electron lifetime, electron transport and charge extraction measurements were performed with a setup previously described. ${ }^{49}$ The bestperformed devices from each dye condition were illuminated with a white LED light source (Luxeon Star $1 \mathrm{~W}$ ) at different intensities.

Photon-induced absorption spectroscopy (PIA). The PIA measurements were performed with a setup described previously. ${ }^{50}$ The samples were excited by illuminating a square-wave modulated blue LED (Luxeon Star $1 \mathrm{~W}$, Royal Blue, $470 \mathrm{~nm}$ ) at an intensity around $8 \mathrm{~mW} \mathrm{~cm}^{-2}$. A white probe light (tungstenhalogen, $20 \mathrm{~W}$ ) irradiated the sample at around $10 \mathrm{~mW} \mathrm{~cm}^{-2}$, which was focused on a SpectraPro-150 monochromator (ACTON) and detected through a UV-enhanced Si photodiode, SR5700 current amplifier and SR830 lock-in amplifier (Stanford Research Systems). Each dye condition was investigated by assembling a transparent sensitized photoanode with a glass slip, infiltrated with the $\mathrm{Cu}^{\mathrm{I} / \mathrm{II}}(\text { tmby })_{2}$ electrolyte or inert electrolyte (=0.1 M LiTFSI and 0.6 M $t$ BP in anhyd. acetonitrile).

Transient absorption spectroscopy (TAS). TAS measurements were conducted with a setup used in previous studies. ${ }^{47} \mathrm{~A}$ frequency-tripled Q-switched Nd:YAG laser and xenon arc lamp 
were coupled with a Surelite OPO Plus optical parametric oscillator (Continuum) to give a pump light energy of $1 \mathrm{~mJ}$ with $13 \mathrm{~ns}$ pulses at $10 \mathrm{~Hz}\left(8 \times 10^{4} \mathrm{~W} \mathrm{~cm}^{-2}\right)$. The sample, a transparent sensitized electrode assembled with a glass slip and infiltrated with $\mathrm{Cu}^{\mathrm{I} / \mathrm{II}}$ (tmby) $)_{2}$ or inert electrolyte, was mounted at a $45^{\circ}$ angle against the light path to provide an active area $\sim 0.35$ $\mathrm{cm}^{2}$. The excitation wavelength was $555 \mathrm{~nm}$ and probe wavelength was $780 \mathrm{~nm}$ for all samples. The response was detected by an LP920 laser flash photolysis/transient absorption spectrometer (Edinburgh Instruments) and recorded on an L900 software.

\section{Results and discussion}

\section{UV-vis absorption spectra}

The XY1 + 5T bath showed a deep red colour as a result of the equimolar mixing of the purple XY1 dye and orange 5T dye. The films immersed in XY1, 5T and XY1 $+5 \mathrm{~T}$ dye baths were all deeply stained, with distinct colours reflecting that of the dye bath (Fig. S2 $\dagger$ ). The UV-vis absorption spectrum of the XY1 + 5Tsensitized $\mathrm{TiO}_{2}$ film has a peak and a shoulder which can be nicely fitted as a superposition of the XY1 and 5T spectra (Fig. 3). XY1 has a shoulder at $443 \mathrm{~nm}$ and an absorption peak at $533 \mathrm{~nm}$, while $5 \mathrm{~T}$ has a peak at $463 \mathrm{~nm}$. The co-sensitized XY1 + $5 \mathrm{~T}$ has a peak at $459 \mathrm{~nm}$ and a shoulder at $534 \mathrm{~nm}$. The peaks assigned to XY1 dye and $5 \mathrm{~T}$ dye are all slightly blue-shifted when

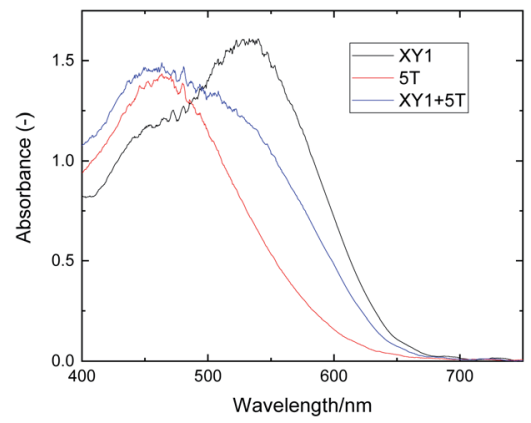

Fig. 3 UV-vis absorption spectra of XY1-, 5T- and XY1 + 5T-sensitized transparent $\mathrm{TiO}_{2}$ films. Baseline was a non-sensitized $\mathrm{TiO}_{2}$ film. compared to the liquid-state absorption. This is a commonly observed phenomenon and is due to the dye-dye or dye- $\mathrm{TiO}_{2}$ interactions in solid-state. ${ }^{51,52}$ Combining the results from visual observation and UV-vis absorption, it can be readily seen that XY1 + 5T sensitized films incorporate both dyes. The loaded dye ratio was further estimated by referencing the solid-state UV-vis absorption data to that in solution. ${ }^{\mathbf{3 8 , 4 1}}$ Full description of the calculation and a range of fittings are specified in the supporting information (Fig. S3 and S4†). The estimation suggests that a 1.7-times greater amount of $5 \mathrm{~T}$ dye in respect to $\mathrm{XY} 1$ dye is incorporated in the XY1 + 5T film. One explanation of this would be the difference in size and geometry of the two dyes; the smaller and more compact 5T dye (MW: 826) has more spatial freedom when binding to the $\mathrm{TiO}_{2}$ surface compared to the bulky XY1 dye (MW: 1539), allowing 5T dye to predominate over XY1 dye when co-sensitized simultaneously.

\section{Photocurrent-voltage properties of the DSSCs}

The $J-V$ curves and basic parameters of the best performing cells are shown in Fig. 4 . The average $J-V$ characteristics of the DSSCs at 1 sun and 0.1 sun are summarized in Table 1.7 cells were fabricated for each dye condition: XY1, 5T and XY1 + 5T. The full statistics are shown as box graphs in Fig. S5 and S6. $\dagger$ The $V_{\text {OC }}$ values at 1 sun marked a high $\sim 1 \mathrm{~V}$ in all cases, which confirms appropriate alignment of the dye HOMO levels and electrolyte redox potential. In particular, an average $V_{\mathrm{OC}}$ of $0.97 \mathrm{~V}(\max .0 .99 \mathrm{~V})$ is the highest value reported for 5Tsensitized DSSCs. ${ }^{\mathbf{4 2}}$ The large enhancement is in good agreement with the redox potential levels of the different electrolytes and our results confirm the importance of selecting the right dye/electrolyte pair. On the other hand, $J_{\mathrm{SC}}$ values of $5 \mathrm{~T}$ cells (avg. $9.9 \mathrm{~mA} \mathrm{~cm}^{-2}$, max. $10.5 \mathrm{~mA} \mathrm{~cm}^{-2}$ ) were lower than the previously reported $\mathrm{I}^{-} / \mathrm{I}_{3}{ }^{-}$or $\mathrm{Co}^{\mathrm{II} / \mathrm{III}}$ systems. ${ }^{42}$ This may be explained by the employment of a thinner $\mathrm{TiO}_{2}$ film $(4 \mu \mathrm{m})$ in this study ( $v s .12 \mu \mathrm{m}$ in literature ${ }^{42}$ ), which will limit the number of dye molecules that can adsorb to the mesoporous $\mathrm{TiO}_{2}$. The co-sensitized XY1 + 5T attained similar PCE to XY1 (avg. 9.1\%) at 1 sun. The best device at 1 sun achieved $\mathrm{PCE}=9.53 \%$, while XY1 and 5T marked 9.56\% and 7.93\%. By lowering the light intensity of the simulated solar spectrum to 0.1 sun, XY1 + 5T (a)

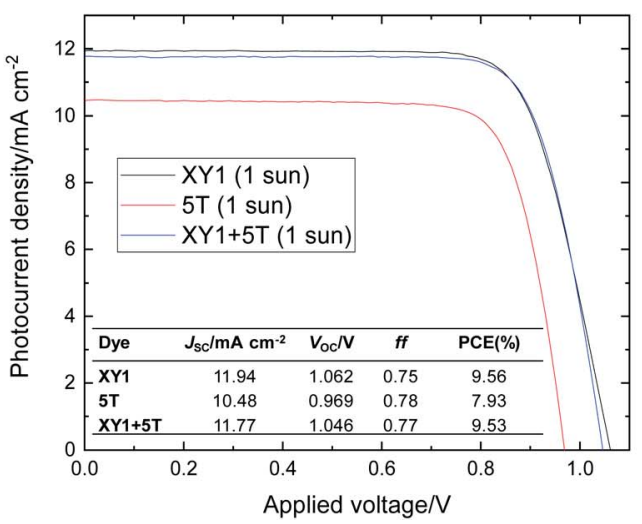

(b)

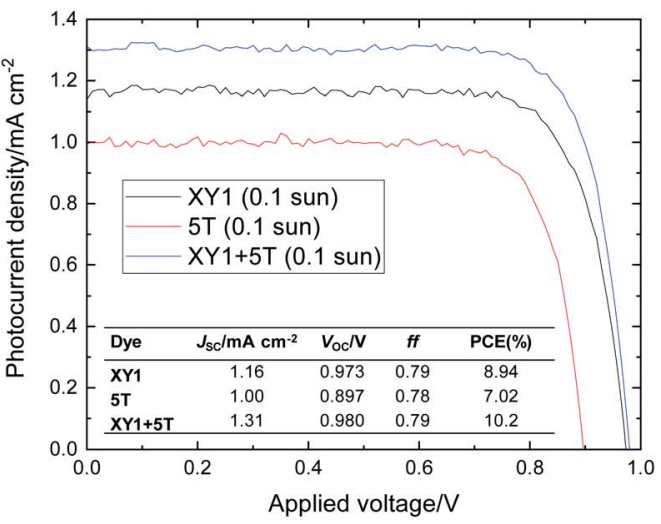

Fig. $4 J-V$ curves of the best DSSCs at (a) 1 sun and (b) 0.1 sun. The insert shows the measured values of the cell parameters. 
Table 1 Statistical performance of DSSCs sensitized with XY1, 5T or a combination of XY1 and $5 T$ under $1 \mathrm{sun}\left(100 \mathrm{~mW} \mathrm{~cm}^{-2}\right)$. The values in brackets are the results under 0.1 sun $\left(10 \mathrm{~mW} \mathrm{~cm}{ }^{-2}\right) .7$ solar cells were fabricated for each dye condition

\begin{tabular}{lllll}
\hline Dye condition & $J_{\mathrm{SC}} / \mathrm{mA} \mathrm{cm}^{-2}$ & $V_{\mathrm{OC}} / \mathrm{V}$ & ff & PCE $(\%)$ \\
\hline $\mathrm{XY} 1$ & $11.4 \pm 0.4(1.2 \pm 0.5)$ & $1.05 \pm 0.01(0.94 \pm 0.04)$ & $0.76 \pm 0.02(0.76 \pm 0.02)$ & $9.1 \pm 0.4(8.6 \pm 0.2)$ \\
$5 \mathrm{~T}$ & $9.9 \pm 0.5(0.96 \pm 0.07)$ & $0.97 \pm 0.01(0.87 \pm 0.03)$ & $0.77 \pm 0.02(0.78 \pm 0.04)$ & $7.5 \pm 0.4(6.5 \pm 0.3)$ \\
$\mathrm{XY} 1+5 \mathrm{~T}$ & $11.8 \pm 0.5(1.25 \pm 0.05)$ & $1.04 \pm 0.01(0.95 \pm 0.03)$ & $0.74 \pm 0.03(0.80 \pm 0.01)$ & $9.1 \pm 0.3(9.4 \pm 0.4)$
\end{tabular}

outperformed XY1 with an average PCE of 9.4\%, compared to XY1 (8.6\%) and 5T (6.5\%). The best XY1 + 5T cell achieved a PCE of $10.2 \%$, which is clearly higher than that of XY1 $(8.94 \%)$ and $5 \mathrm{~T}(7.02 \%)$. The improved performance of $\mathrm{XY} 1+5 \mathrm{~T}$ at 0.1 sun may be related to the equilibrium drift between mass transport and charge recombination in the electrolyte and at the electrolyte $/ \mathrm{TiO}_{2}$ surface.

\section{Incident photon-to-current efficiency}

The IPCE spectra of the DSSCs and the integrated $J_{\mathrm{SC}}$ values are summarized in Fig. 5. XY1 and 5T have a maximum IPCE around $580 \mathrm{~nm}$ and $420 \mathrm{~nm}$, respectively. The two dyes complement each other in the case of XY1 + 5T to maintain an IPCE $>65 \%$ throughout $400-620 \mathrm{~nm}$. The integrated $J_{\mathrm{SC}}$ values at $9.7 \mathrm{~mA} \mathrm{~cm}^{-2}$ for XY1, $6.9 \mathrm{~mA} \mathrm{~cm}^{-2}$ for $5 \mathrm{~T}$ and $9.6 \mathrm{~mA} \mathrm{~cm}^{-2}$ for $\mathrm{XY} 1+5 \mathrm{~T}$ correlate well with the $J_{\mathrm{SC}}$ obtained from the $J-V$ measurements.

\section{Time-resolved 'toolbox' measurements}

Time-resolved 'toolbox' measurements were carried out for the best solar cells with each dye condition, to investigate the relation between each dye condition and charge transport behaviour in the devices. The $V_{\mathrm{OC}}$ of an illuminated cell is defined by:

$$
V_{\mathrm{OC}}=E_{\mathrm{F}}-E_{0}
$$

where $E_{\mathrm{F}}$ is the quasi Fermi energy level of the $\mathrm{TiO}_{2}$ network and $E_{0}$ is the redox potential of the electrolyte. $E_{\mathrm{F}}$ is then given by:

$$
E_{\mathrm{F}}=E_{\mathrm{C}}+k T \ln \left(n_{\mathrm{c}} / N_{\mathrm{c}}\right)
$$

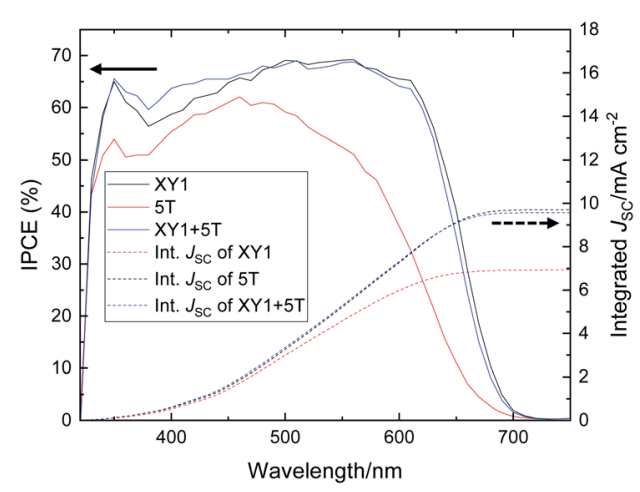

Fig. 5 IPCE curves of XY1 (black), $5 T$ (red) and XY1 + 5T (blue)sensitized DSSCs. The dotted lines are the integrated current density values. where $E_{\mathrm{C}}$ is the conduction band energy, $k T$ is the thermal energy (25.7 meV at r.t.), $n_{\mathrm{c}}$ is the density of conduction band electrons and $N_{\mathrm{c}}$ is the density of conduction band states. ${ }^{\mathbf{4 9}}$ From eqn (1) and (2), one can tell that $E_{\mathrm{F}}$ increases proportionally with $\ln \left(n_{\mathrm{c}}\right)$, while $V_{\mathrm{OC}}$ increases along with $E_{\mathrm{F}}$. The semi logarithmic trend of the extracted charge $\left(Q_{\text {ext }}\right)$ under opencircuit conditions (Fig. S7 $\dagger$ ) gives information on the conduction band edge shifts due to the binding of the dye to $\mathrm{TiO}_{2}$. Larger $Q_{\text {ext }}$ values relate to higher $n_{\mathrm{c}}$. At a given $Q_{\text {ext }}$ (or $n_{\mathrm{c}}$ ), the $V_{\mathrm{OC}}$ of XY1 $+5 \mathrm{~T}$ is $0.039 \mathrm{~V}$ smaller than that of XY1. However, the actual voltage difference is negligible compared to this value (Fig. 4, Table 1). This indicates that the recombination properties are more significant to understand the cell performance. To note, the conduction band shift between $5 \mathrm{~T}$ and $\mathrm{XY} 1+5 \mathrm{~T}$ is even smaller.

Fig. 6 depicts the electron lifetime $\left(\tau_{\mathrm{e}}\right)$ under open-circuit conditions. The electron lifetime refers to the duration of the conduction band electrons before they recombine with the $\mathrm{Cu}^{2+}$ species. All curves show an exponential decrease, with almost identical slopes for $5 \mathrm{~T}$ and $\mathrm{XY} 1+5 \mathrm{~T}\left(-9.02 \mathrm{~s} \mathrm{~V}^{-1},-10.6 \mathrm{~s} \mathrm{~V}^{-1}\right)$ and a slightly deviated slope for XY1 $\left(-7.39 \mathrm{~s} \mathrm{~V}^{-1}\right.$ in the linear region). $\mathrm{XY} 1+5 \mathrm{~T}$ showing similar $\tau_{\mathrm{e}}$ values to $\mathrm{XY} 1$ around the $V_{\mathrm{OC}} \sim 1.05$ range in Fig. 6 explains the similar and high $V_{\mathrm{OC}}(>1.0$ V) achieved by both dye conditions at 1 sun (Table 1). The recombination rates are likely to be similar around this intensity. The less inclined $\tau_{\mathrm{e}} v s . V_{\mathrm{OC}}$ (or $\tau_{\mathrm{e}} v s$. light intensity) slope with XY1 + 5T compared to XY1 suggests that the $\tau_{\mathrm{e}}$ of XY1 $+5 \mathrm{~T}$ is likely to exceed that of XY1 at lower light intensities (by extrapolating the XY1 curve towards smaller $\left.V_{\mathrm{OC}}\right)$. Indeed, the average

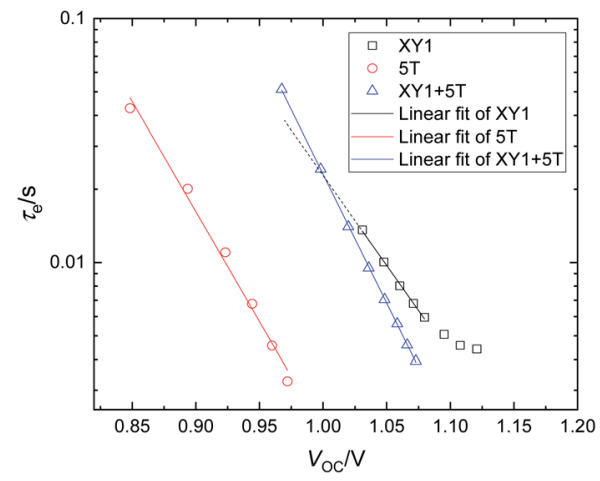

Fig. 6 Electron lifetime $\left(\tau_{\mathrm{e}}\right)$ of the best DSSCs (XY1: black square; $5 T$ : red circle; $X Y 1+5 T$ : blue triangle), plotted against $V_{O C}$ at different light intensities. The solid lines are linear fits of the linear region. The dashed line for $X Y 1$ is an extension of the linear fit, which intercepts the linear fit of $\mathrm{XY} 1+5 \mathrm{~T}$ at $\mathrm{V}_{\mathrm{OC}} \sim 1.0016 \mathrm{~V}$. 
$V_{\mathrm{OC}}$ trend of $\mathrm{XY} 1$ and $\mathrm{XY} 1+5 \mathrm{~T}$ is flipped over from 1 sun to 0.1 sun, although the difference is very small. Investigation of the $\mathrm{XY} 1+5 \mathrm{~T}$ cell performance at low light conditions may give further indications on this. The $\mathrm{D}-\mathrm{A}-\pi-\mathrm{A}$ dye is designed so that the bulky donor prevents the $\mathrm{Cu}^{2+}$ ions from approaching the $\mathrm{TiO}_{2}$ surface and removing electrons from the $\mathrm{TiO}_{2}$ conduction band. As such, the improved electron lifetime of XY1 + 5T indicates that the amount of XY1 contained in $\mathrm{XY} 1+5 \mathrm{~T}$ is sufficient to suppress recombination with the $\mathrm{Cu}^{2+}$ species. ${ }^{38}$ The improved coverage of the mesoporous $\mathrm{TiO}_{2}$ with the two dyes may also suppress recombination sites in general. The size/ shape difference between $5 \mathrm{~T}$ and XY1 is probably beneficial to reach optimum coverage with minimum aggregation (Fig. S8(a)) $+\dagger$

The electron transport time is plotted against $J_{\mathrm{SC}}$ (Fig. S9†) to investigate the speed of charge transport within the $\mathrm{TiO}_{2}$ network upon illumination. Interestingly, the transport speed declines (larger $\tau_{\mathrm{tr}}$ ) when the two dyes are combined. Slower transport during operation may generally lead to more recombination, however this is not the case with $\mathrm{XY} 1+5 \mathrm{~T}$ according to the electron lifetime trends. The change in transport time might be due to some surface modification of the $\mathrm{TiO}_{2}$ caused by the adsorption of both dyes. The increased risk for the electrons to recombine with $\mathrm{Cu}^{2+}$ is however likely to be traded off if the dye coverage is higher and the number of recombination sites is reduced. When both $\mathrm{XY} 1$ and $5 \mathrm{~T}$ dye are present during the sensitization step, some of the voids left on the $\mathrm{TiO}_{2}$ surface by the bulky Y-shape XY1 dye could be occupied by the compact Ishape 5T dye (Fig. S8(b)). $\dagger$ This will then lead to an increased number of adsorbed dye molecules in XY1 + 5T compared to XY1.

\section{Insights on charge transport by absorption spectroscopy}

The PIA spectra of the sensitized $\mathrm{TiO}_{2}$ films for each dye condition (Fig. 7(a)) also give an insight about the electron transport and recombination of an operating device. The sensitized films in contact with an inert electrolyte show a ground-state dye bleach at $\sim 630 \mathrm{~nm}, \sim 590 \mathrm{~nm}$ and $\sim 610 \mathrm{~nm}$ for XY1, 5T and XY1 + 5T, respectively. In addition, a broader absorption peak is seen at $\sim 790 \mathrm{~nm}$ for $5 \mathrm{~T}$ and $>900 \mathrm{~nm}$ for XY1 and $\mathrm{XY} 1+5 \mathrm{~T}$. Inclusion of $\mathrm{Cu}^{\mathrm{I} / \mathrm{II}}(\mathrm{tmby})_{2}$ in the electrolyte drastically changes the spectral features. Complete disappearance of the broad absorption indicates the efficient regeneration of the dye molecules by the $\mathrm{Cu}^{\mathrm{I} / \mathrm{II}}(\mathrm{tmby})_{2}$ couple in all dye conditions. The prominent dip in the range of 500-700 $\mathrm{nm}$ can be assigned to a Stark bleach, ${ }^{53}$ where peaks are observed at $630 \mathrm{~nm}(\mathrm{XY} 1), \sim 590 \mathrm{~nm}(5 \mathrm{~T})$ and $620 \mathrm{~nm}(\mathrm{XY} 1+5 \mathrm{~T})$, matching well with the bleach profiles in the inert condition. The highest bleach amplitude of $\mathrm{XY} 1+5 \mathrm{~T}$ in both inert and redox-active conditions may suggest higher dye coverage of XY1 $+5 \mathrm{~T}$ compared to XY1 or $5 \mathrm{~T}$, consistent with our earlier predictions.

TAS results are shown in Fig. 7(b). The absorption decays were fitted with an extended exponential to estimate the decay half-time for each condition. The half-time derived from the inert system relates to the recombination time of the conduction band electrons with the oxidized dyes, while that from the $\mathrm{Cu}^{\mathrm{I} / \mathrm{II}}(\mathrm{tmby})_{2}$ system relates to the speed of dye regeneration by the redox couple. The regeneration efficiency is calculated from the following equation:

$$
\phi_{\text {reg }}=1-\left(\tau_{1 / 2, \text { reg }} / \tau_{1 / 2, \text { rec }}\right)
$$

where $\tau_{1 / 2 \text {,reg }}$ is the regeneration half-time and $\tau_{1 / 2 \text {,rec }}$ is the recombination half-time. ${ }^{47,50}$ The recombination of $5 \mathrm{~T}(247 \mu \mathrm{s})$ was found to be 6-fold slower compared to XY1 (40 $\mu \mathrm{s})$. This extremely slow charge recombination with the oxidized $5 \mathrm{~T}$ is interesting as the $5 \mathrm{~T}$ has a deeper HOMO level than XY1, which would normally make it easier to recombine due to the larger driving force. The trend may be due to the $\pi-\mathrm{A}$ structure of $5 \mathrm{~T}$, i.e. lack of a proper donor moiety, an uncommon feature in most dye designs. In addition, $5 \mathrm{~T}$ shows a 3-fold faster regeneration compared to XY1 $(0.7 \mu \mathrm{s} v s .1 .9 \mu \mathrm{s})$ to achieve an overall regeneration efficiency of $99.7 \%$. The profile of XY1 $+5 \mathrm{~T}$ is (a)

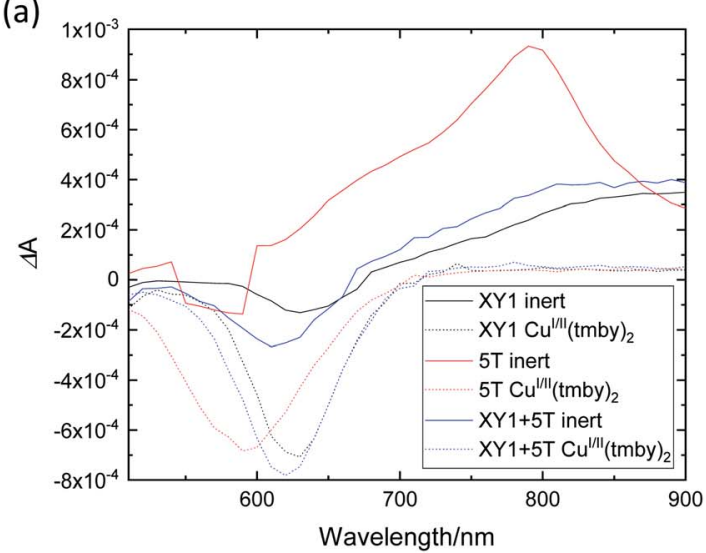

(b)

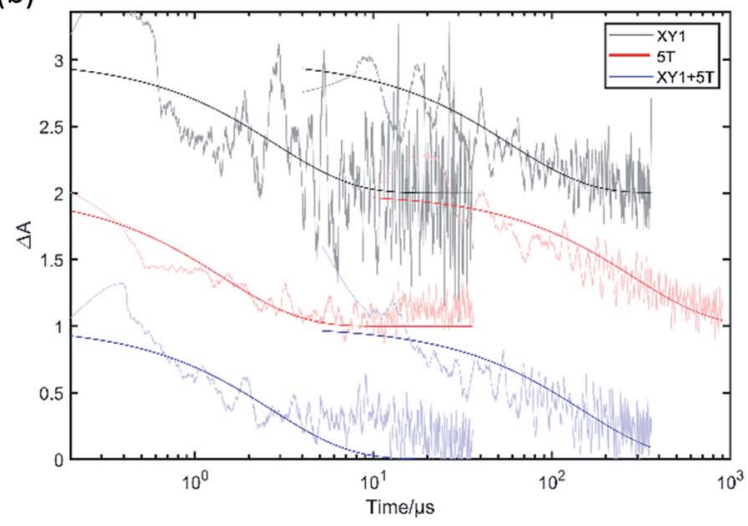

Fig. 7 (a) PIA curves of the sensitized transparent $\mathrm{TiO}_{2}$ films combined with an inert electrolyte (LiTFSI and tBP in acetonitrile, solid line) or Cu' "(tmby) $)_{2}$ electrolyte(dotted line). (b) TAS curves of the samples used in PIA measurements, with the right-hand curves corresponding to inert samples and left-hand curves corresponding to the samples with $\mathrm{Cu}^{1 / 1}(\mathrm{tmby})_{2}$. The solid exponential lines in the TAS spectra are the fitted decay lines to calculate the regeneration efficiency. 
closer to the trend of XY1 but slightly improved for both $\tau_{1 / 2 \text {,rec }}$ $(45 \mu \mathrm{s})$ and $\tau_{1 / 2 \text {,reg }}(1.7 \mu \mathrm{s})$, resulting in $\phi_{\text {reg }}=96 \%$.

\section{Potential of XY1 + 5T for indoor DSSCs}

With the contribution of both dyes in XY1 + 5T DSSCs confirmed, it is reasonable to assess their potential as indoor-operational devices; since this is the most promising direction for DSSCs in practice. In fact, the superior performance of XY1 + 5T to XY1 at 0.1 sun ( $\max . \mathrm{PCE}=10.2 \%)$ indicates that this dye combination may work efficiently at ultra-low light intensities. The device fabrication and testing were similar to the work reported by Freitag et al. ${ }^{8}$ In brief, the active area was made larger $\left(3.20 \mathrm{~cm}^{2}\right)$ than typical lab devices $\left(0.160 \mathrm{~cm}^{2}\right)$ to follow conditions closer to real-life applications. A warm white fluorescent lamp at 1000 lux (303.1 $\mu \mathrm{W} \mathrm{cm}{ }^{-2}$ ) was chosen as the source. The PCE was calculated by $P_{\text {out }} / P_{\text {in }}$, where $P_{\text {in }}$ is the incident light intensity (i.e. 303.1 $\mu \mathrm{W} \mathrm{cm}{ }^{-2}$ in this study) and $P_{\text {out }}$ is the maximum power density recorded by the cell. Fig. 8 shows the $J-V$ curve of the XY1 +5 T cell tested by this condition. A PCE of $29.2 \%$ was achieved, which is clearly higher than any other established photovoltaic technology including silicon and thin-films, and even amongst the highest achieved with DSSCs tested under similar conditions. ${ }^{6-9,54}$ Another DSSC was fabricated and tested to confirm the reproducibility, where the slightly lower ff led to an avg. PCE of $28 \%$ with the 2 cells (Fig. S10, $\dagger$ Table 1 ). The 3 -fold efficiency jump from 1 sun to indoor-light originates from close matching of the absorption range of the dyes to the input spectral range of the light source. Although the overall PCE is lower than the best reported PCE of $31.8 \%,{ }^{9}$ the $V_{\mathrm{OC}}$ is comparable, marking $0.86 \mathrm{~V}$ in average. The relatively high $V_{\mathrm{OC}}$ and PCE under ultra-low light intensity (i.e. 1000 lux) supports our findings from the electron lifetime studies. The current output with these XY1 $+5 \mathrm{~T}$ cells is over $100 \mu \mathrm{A} \mathrm{cm}^{-2}$, which is the standard power required for lowpower electronic devices in sleeping mode. ${ }^{8}$ These preliminary results suggest that the $3.2 \mathrm{~cm}^{2} \mathrm{XY} 1+5 \mathrm{~T}$ DSSC is a practical device from the performance perspective.

In practice, the long-term stability of the co-sensitized devices should also be taken into consideration alongside their performance and cost. DSSCs are known to be generally chemically/ thermally stable and are tolerant to moisture or oxygen at

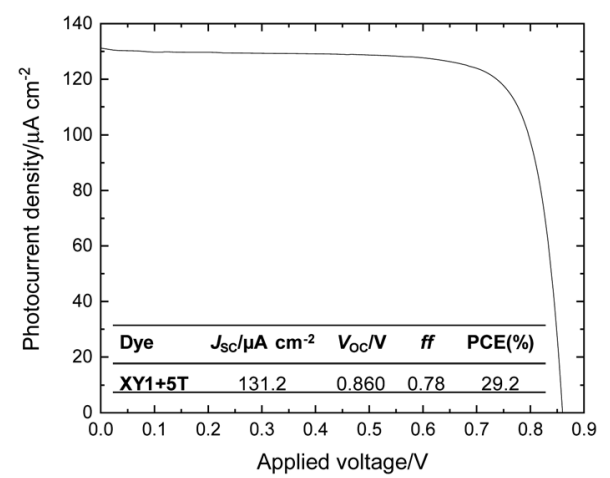

Fig. $8 \mathrm{~J}-V$ curve of the best XY1 $+5 T$ DSSC at 1000 lux. The inset shows the measured values of the cell parameters. ambient levels. ${ }^{55,56}$ However, their performance can slowly deteriorate due to leakage of the volatile electrolyte from the imperfections of the sealing of the cell. In this sense, proper encapsulation of the device is crucial for the liquid electrolyte cell. On the other hand, the $\mathrm{Cu}^{\mathrm{I} / \mathrm{II}}(\mathrm{tmby})_{2}$ electrolyte selected in this study is a specific type of electrolyte that steadily converts into a stable solid hole conductor by controlled evaporation of the solvent. ${ }^{9,45,46,48}$ The device architecture employed in this study is therefore expected to facilitate future assessments on the longterm stability of the co-sensitized DSSCs as solid-state cells.

\section{Cost estimation}

Finally, the performance of the DSSCs sensitized by XY1, $5 \mathrm{~T}$ and $\mathrm{XY} 1+5 \mathrm{~T}$ is discussed in relation to their preparation cost. The costs were calculated following a simple approach presented by Maciejczyk et al. In brief, the cost of XY1 and 5T was estimated from the cost of their synthesis, and the synthesis cost was approximated to the cost of the starting materials. ${ }^{57}$ We believe that this assumption is valid, since the cost of the work-up depends heavily on the scale of the synthesis and becomes negligible especially at industrial scale. The calculations in full detail are shown in Fig. S1 and S11. $\dagger$

The molar equivalent of the adsorbed dye(s) in XY1, 5T and $\mathrm{XY} 1+5 \mathrm{~T}$ was calculated by referencing the solid-state UV-vis spectra of the dyes to their liquid-state UV-vis spectra, where the peak absorbance was compared. The ratio of XY1 and $5 \mathrm{~T}$ in the co-sensitized film was estimated by fitting the spectra of XY1 $+5 \mathrm{~T}$ to a sum of XY1 and 5T at different ratios. The fitted curves and error trend are described in Fig. S3 and S4. $\dagger$

From the cost calculation of each dye and estimated molar equivalent of each dye in XY1, 5T and XY1 + 5T, we have found that the cost of dye per unit area can be reduced to $c a$. $70 \%$ if we blend 5T with XY1 to reach a performance similar to XY1 (avg. PCE = $9.1 \%$ at 1 sun) (Table $\mathrm{S} 2 \dagger$ ). The contribution comes from the much cheaper 5T dye ( $\$ 275$ per mmol), whose synthesis cost is $c a .70 \%$ less than that of XY1 dye (\$868 per mmol). Although the number of $5 \mathrm{~T}$ dye molecules in a 5T-only device is estimated to be more than the number of XY1 dye molecules in an XY1 device $(1.29 \mathrm{~mol}$ eq. vs. $1 \mathrm{~mol}$ eq.), the total dye cost per unit area is $c a .60 \%$ less in 5T. Likewise, our estimate shows that XY1 + 5T contains 1.7-times more 5T dye than XY1 dye, with a total of 1.3 mol equivalent XY1 dye and 5T dye. The estimation is in good accord with the previous suggestions that XY1 + 5T-sensitized films have higher dye coverage. When we recalculate the costs per unit area as "cost per unit area per PCE" (=cost performance), the sensitizer cost of XY1 $+5 \mathrm{~T}$ decreases from $74 \%$ to $68 \%$ in respect to the cost of XY1-only with 0.1 sun illumination. The value is relatively close to the cost performance of $5 \mathrm{~T}$-only $(54 \%)$. The results demonstrate that $5 \mathrm{~T}$ dye is indeed a good co-sensitizer for XY1 dye not only in terms of performance but also in terms of cost.

\section{Co-sensitization as a cost-improvement strategy}

Our results show that the co-sensitization effectively improves parameters such as the optical absorption/IPCE, electron lifetime, dye regeneration, dye coverage and cost performance compared to the mono-sensitized DSSCs. In this study, we 
adopted a simple $1: 1(\mathrm{~mol} / \mathrm{mol})$ dye mixing ratio of XY1 and 5T in order to make our findings applicable to a broader choice of dye conditions. For instance, fine tuning of the dye mixing ratio has been investigated by Freitag et al. ${ }^{8}$ where the best performance was achieved with a ratio of D35 + XY1 $=4: 1$. In their study, the $1: 1$ ratio is shown to be very close to the optimized $4: 1$ ratio. In this view, we believe that the present study provides sufficient general demonstration of co-sensitization for cost-performance improvement purposes. In the case of $\mathrm{XY} 1+5 \mathrm{~T}$, our data suggest that the binding ratio of XY1 dye and $5 \mathrm{~T}$ dye in $\mathrm{XY} 1+5 \mathrm{~T}$ is $\sim 5: 8$ when a XY1 $: 5 \mathrm{~T}=1: 1(\mathrm{~mol} / \mathrm{mol})$ bath is used. We could thus predict that increasing the amount of XY1 in the dye bath would perhaps lead to extended absorption in the longer wavelength region (as in Fig. S3, $\uparrow 0.7$ $\mathrm{XY} 1+0.55 \mathrm{~T})$ and enhance the electron lifetime, resulting in higher PCE. On the other hand, increasing the amount of 5T will steadily reduce the total dye cost and also improve the dye coverage, which might lead to even cheaper DSSCs with maintained performance. Since the cost-performance is based on the balance of cost and performance, the optimized dye mixing ratio should be investigated experimentally by comparing the PCE and optical absorption for each dye ratio.

\section{Conclusions}

A less expensive $\pi-\mathrm{A} 5 \mathrm{~T}$ dye was co-sensitized with a betterperforming $\mathrm{D}-\mathrm{A}-\pi-\mathrm{A}$ XY1 dye to achieve efficient $\mathrm{Cu}^{\mathrm{I} /}$ ${ }^{\text {II }}$ (tmby) $)_{2}$-based DSSCs. Photophysical measurements revealed the complementary effects of XY1 and 5T in the co-sensitized devices during operation. DSSCs incorporating both XY1 and 5T achieved a PCE of 9.5\% (avg. 9.1\%) at 1 sun and $10.2 \%$ (avg. $9.4 \%$ ) at 0.1 sun, which is similar or superior to the DSSCs using only XY1. The XY1 + 5T was further tested in indoor-light conditions, where the best cell reached an impressive power conversion efficiency of $29.2 \%$, which is among the highest achieved by any reported solar cells under a similar condition. The co-sensitized XY1 + 5T solar cells are also less expensive than the XY1-only cells, which makes them more commercially viable than the relatively high performing mono-sensitized solar cells in terms of cost and performance.

We hope this study will shed light on another aspect of the simple and well-known co-sensitization technique, with some useful indications on how to select the appropriate combination of dyes and how to evaluate them when aiming for practical usage. Overall, in addition to the established efficiency enhancement, we have demonstrated that the co-sensitization approach has great potential to be more widely studied as a cost-reduction strategy to develop genuinely practical DSSCs.

\section{Conflicts of interest}

There are no conflicts to declare.

\section{Acknowledgements}

The authors thank Dr Miquel Planells for providing the 5T dye. E. T. thanks the Energy Technology Partnership for their funding towards collaborative research and JASSO for their PhD studentship.

\section{Notes and references}

1 World Energy Council, World Energy Resources 2016, London, United Kingdom, 2016.

2 R. G. Miller and S. R. Sorrell, Philos. Trans. R. Soc., A, 2014, 372, 20130179.

3 United Nations, World Population Prospects 2019, New York, United States of America, 2019.

4 K. Rajkanan, R. Singh and J. Shewchun, Solid-State Electron., 1979, 22, 793-795.

5 K. L. Chopra, P. D. Paulson and V. Dutta, Prog. Photovolt. Res. Appl., 2004, 12, 69-92.

6 N. H. Reich, W. G. J. H. M. van Sark and W. C. Turkenburg, Renewable Energy, 2011, 36, 642-647.

7 C. Hora, F. Santos, M. G. F. Sales, D. Ivanou and A. Mendes, ACS Sustainable Chem. Eng., 2019, 7, 13464-13470.

8 M. Freitag, J. Teuscher, Y. Saygili, X. Zhang, F. Giordano, P. Liska, J. Hua, S. M. Zakeeruddin, J. Moser, M. Grätzel and A. Hagfeldt, Nat. Photonics, 2017, 11, 372-378.

9 Y. Cao, Y. Liu, S. M. Zakeeruddin, A. Hagfeldt and M. Grätzel, Joule, 2018, 2, 1108-1117.

10 C. Sima, C. Grigoriu and S. Antohe, Thin Solid Films, 2010, 519, 595-597.

11 T. Kawashima, T. Ezure, K. Okada, H. Matsui, K. Goto and N. Tanabe, J. Photochem. Photobiol., A, 2004, 164, 199-202.

12 V. Kumar, N. Singh, V. Kumar, L. P. Purohit, A. Kapoor, O. M. Ntwaeaborwa and H. C. Swart, J. Appl. Phys., 2013, 114, 134506.

13 K. Yoo, J.-Y. Kim, J. A. Lee, J. S. Kim, D.-K. Lee, K. Kim, J. Y. Kim, B. Kim, H. Kim, W. M. Kim, J. H. Kim and M. J. Ko, ACS Nano, 2015, 9, 3760-3771.

14 X. Lü, X. Mou, J. Wu, D. Zhang, L. Zhang, F. Huang, F. Xu and S. Huang, Adv. Funct. Mater., 2010, 20, 509-515.

15 P. Tiwana, P. Docampo, M. B. Johnston, H. J. Snaith and L. M. Herz, ACS Nano, 2011, 5, 5158-5166.

16 E. Tanaka, L. Nurdiwijayanto, M. Hagiwara and S. Fujihara, J. Solid State Electrochem., 2018, 22, 3119-3127.

17 S. Muduli, O. Game, V. Dhas, K. Vijayamohanan, K. A. Bogle, N. Valanoor and S. B. Ogale, Sol. Energy, 2012, 86, 1428-1434.

18 Y. Jo, C. L. Jung, J. Lim, B. H. Kim, C. H. Han, J. Kim, S. Kim, D. Kim and Y. Jun, Electrochim. Acta, 2012, 66, 121-125.

19 S. Ito, H. Miura, S. Uchida, M. Takata, K. Sumioka, P. Liska, P. Comte, P. Péchy and M. Grätzel, Chem. Commun., 2008, 5194-5196.

20 K. Kakiage, Y. Aoyama, T. Yano, K. Oya, J. Fujisawa and M. Hanaya, Chem. Commun., 2015, 51, 15894-15897.

21 A. Abbotto, N. Manfredi, C. Marinzi, F. De Angelis, E. Mosconi, J. Yum, Z. Xianxi, K. Nazeeruddin and M. Grätzel, Energy Environ. Sci., 2009, 2, 1094-1101.

22 G. Boschloo and A. Hagfeldt, Acc. Chem. Res., 2009, 42, 18191826.

23 A. Yella, H.-W. Lee, H. N. Tsao, C. Yi, A. K. Chandiran, M. K. Nazeeruddin, E. W.-G. Diau, C.-Y. Yeh, 
S. M. Zakeeruddin and M. Grätzel, Science, 2011, 334, 629634.

24 M. Freitag, F. Giordano, W. Yang, M. Pazoki, Y. Hao, B. Zietz, M. Grätzel, A. Hagfeldt and G. Boschloo, J. Phys. Chem. C, 2016, 120, 9595-9603.

25 M. Wang, N. Chamberland, M. Grätzel, L. Breau, J.-E. Moser, R. Humphry-Baker, B. Marsan and S. M. Zakeeruddin, Nat. Chem., 2010, 2, 385-389.

26 X. Meng, C. Yu, X. Song, Y. Liu, S. Liang, Z. Liu, C. Hao and J. Qiu, Adv. Energy Mater., 2015, 5, 1500180.

27 J. S. Jang, D. J. Ham, E. Ramasamy, J. Lee and J. S. Lee, Chem. Commun., 2010, 46, 8600-8602.

28 J. Wu, Q. Li, L. Fan, Z. Lan, P. Li, J. Lin and S. Hao, J. Power Sources, 2008, 181, 172-176.

29 H. Ellis, N. Vlachopoulos, L. Häggman, C. Perruchot, M. Jouini, G. Boschloo and A. Hagfeldt, Electrochim. Acta, 2013, 107, 45-51.

30 M. Ye, X. Wen, M. Wang, J. Iocozzia, N. Zhang, C. Lin and Z. Lin, Mater. Today, 2015, 18, 155-162.

31 S. Mathew, A. Yella, P. Gao, R. Humphry-Baker, B. F. E. Curchod, N. Ashari-Astani, I. Tavernelli, U. Rothlisberger, M. K. Nazeeruddin and M. Grätzel, Nat. Chem., 2014, 6, 242-247.

32 J. M. Cole, G. Pepe, O. K. Al Bahri and C. B. Cooper, Chem. Rev., 2019, 119, 7279-7327.

33 J. Fang, H. Mao, J. Wu, X. Zhang and Z. Lu, Appl. Surf. Sci., 1997, 119, 237-241.

34 E. A. Knyazeva, W. Wu, T. N. Chmovzh, N. Robertson, J. D. Woollins and O. A. Rakitin, Sol. Energy, 2017, 144, 134-143.

35 M. Kimura, H. Nomoto, N. Masaki and S. Mori, Angew. Chem., Int. Ed., 2012, 51, 4371-4374.

36 J. Liu, Y. Wu, C. Qin, X. Yang, T. Yasuda, A. Islam, K. Zhang, W. Peng, W. Chen and L. Han, Energy Environ. Sci., 2014, 7, 2963-2967.

37 A. Islam, M. Akhtaruzzaman, T. H. Chowdhury, C. Qin, L. Han, I. M. Bedja, R. Stalder, K. S. Schanze and J. R. Reynolds, ACS Appl. Mater. Interfaces, 2016, 8, 46164623.

38 X. Zhang, Y. Xu, F. Giordano, M. Schreier, N. Pellet, Y. Hu, C. Yi, N. Robertson, J. Hua, S. M. Zakeeruddin, H. Tian and M. Grätzel, J. Am. Chem. Soc., 2016, 138, 10742-10745.

39 M. Planells, A. Abate, H. J. Snaith and N. Robertson, ACS Appl. Mater. Interfaces, 2014, 6, 17226-17235.

40 A. Abate, M. Planells, D. J. Hollman, S. D. Stranks, A. Petrozza, A. R. S. Kandada, Y. Vaynzof, S. K. Pathak, N. Robertson and H. J. Snaith, Adv. Energy Mater., 2014, 4, 1400166.
41 Y. Hu, A. Ivaturi, M. Planells, C. L. Boldrini, A. O. Biroli and N. Robertson, J. Mater. Chem. A, 2016, 4, 2509-2516.

42 Y. Hu, A. Abate, Y. Cao, A. Ivaturi, S. M. Zakeeruddin, M. Grätzel and N. Robertson, J. Phys. Chem. C, 2016, 120, 15027-15034.

43 Merck Price Catalogue, https:/www.sigmaaldrich.com/ catalog/https://www.sigmaaldrich.com/catalog/product/

aldrich/703214 lang $=$ en\&region $=\mathrm{GB}, \quad$ accessed $\quad 20$ September 2019.

44 Y. Saygili, M. Söderberg, N. Pellet, F. Giordano, Y. Cao, A. B. Muñoz-García, S. M. Zakeeruddin, N. Vlachopoulos, M. Pavone, G. Boschloo, L. Kavan, J.-E. Moser, M. Grätzel, A. Hagfeldt and M. Freitag, J. Am. Chem. Soc., 2016, 138, 15087-15096.

45 M. Freitag, Q. Daniel, M. Pazoki, K. Sveinbjörnsson, J. Zhang, L. Sun, A. Hagfeldt and G. Boschloo, Energy Environ. Sci., 2015, 8, 2634-2637.

46 W. Zhang, Y. Wu, H. W. Bahng, Y. Cao, C. Yi, Y. Saygili, J. Luo, Y. Liu, L. Kavan, J. E. Moser, A. Hagfeldt, H. Tian, S. M. Zakeeruddin, W.-H. Zhu and M. Grätzel, Energy Environ. Sci., 2018, 11, 1779-1787.

47 H. Michaels, I. Benesperi, T. Edvinsson, A. Belén MuñozGarcia, M. Pavone, G. Boschloo and M. Freitag, Inorganics, 2018, 6, 53.

48 Y. Cao, Y. Saygili, A. Ummadisingu, J. Teuscher, J. Luo, N. Pellet, F. Giordano, S. M. Zakeeruddin, J.-E. Moser, M. Freitag, A. Hagfeldt and M. Grätzel, Nat. Commun., 2017, 8, 15390.

49 G. Boschloo, L. Häggman and A. Hagfeldt, J. Phys. Chem. B, 2006, 110, 13144-13150.

50 G. Boschloo and A. Hagfeldt, Inorg. Chim. Acta, 2008, 361, 729-734.

51 L. Ducasse, F. Castet, R. Méreau, S. Nénon, J. Idé, T. Toupance and C. Olivier, Chem. Phys. Lett., 2013, 556, 151-157.

52 W. Zhu, Y. Wu, S. Wang, W. Li, X. Li, J. Chen, Z.-S. Wang and H. Tian, Adv. Funct. Mater., 2011, 21, 756-763.

53 M. Pazoki, A. Hagfeldt and G. Boschloo, Electrochim. Acta, 2015, 179, 174-178.

54 M. Foti, C. Tringali, A. Battaglia, N. Sparta, S. Lombardo and C. Gerardi, Sol. Energy Mater. Sol. Cells, 2014, 130, 490-494. 55 H. Matsui, K. Okada, T. Kitamura and N. Tanabe, Sol. Energy Mater. Sol. Cells, 2009, 93, 1110-1115.

56 K. Zhu, S.-R. Jang and A. J. Frank, Energy Environ. Sci., 2012, 5, 9492-9495.

57 M. R. Maciejczyk, R. Chen, A. Brown, N. Zheng and N. Robertson, J. Mater. Chem. C, 2019, 7, 8593-8598. 